\title{
Does Exist A Correlation Between BMI and Gleason Patterns 4 and 5 At Prostate Biopsy?
}

Vincenzo Serretta ${ }^{1 *}$, Giovanni Caruana ${ }^{1}$, Francesco Sommatino $^{1}$, Salvatore Scurria ${ }^{1}$, Giuseppe Carità $^{1}$, Luigi Vaccarella ${ }^{1}$, Federico Torretta $^{2}$, Giuseppe Cicero ${ }^{3}$, Marcello Daricello ${ }^{4}$, Vito Franco ${ }^{5}$

${ }^{1}$ Department of Surgical, Oncological and Stomathological Sciences, Section of Urology, University of Palermo, Italy

${ }^{2}$ Department of Economics, Business and Statistics, University of Palermo, Italy

${ }^{3}$ Department of Surgical, Oncological and Stomathological Sciences, Section of Clinical Oncology, University of Palermo, Italy

${ }^{4}$ Department of Biochemical Sciences, University of Palermo, Italy

${ }^{5}$ Department of Sciences for Health Promotion and Maternal and Child "G. D'Alessandro" -Section of Pathology-, University of Palermo, Italy

\begin{abstract}
Background: Obesity has been related with higher Gleason grade and worse prognosis. Increasing proportion of Gleason pattern 4 or 5 is a critical factor for biochemical recurrence, progression and mortality. The endocrine activity of visceral fat could be responsible of the differentation of the prostatic malignant cell towards a more aggressive fenotype. The aim of our study was to correlate Body Mass Index with the presence of Gleason pattern 4 or higher at biopsy.

Materials and Methods: Consecutive patients with positive prostate biopsy were included. A transrectal prostate biopsy procedure with 12 cores, was performed. All tissue samples were reviewed.

Results: Out of 135 patients diagnosed with prostate cancer at biopsy, a Gleason. pattern 4 or 5 was evident in 57 (42\%) patients, while it was not detected in $78(58 \%)$. The statistical analysis did not demonstrate a correlation between the histological expression of Gleason pattern 4 or 5 in the bioptic specimens and the BMI class. Conclusion: Although high risk prostate cancer has been reported more frequent in patients with higher BMI, in our experience, no significant correlation between BMI and Gleason patterns 4 and 5 at biopsy was detected. Other factors responsible for the worse prognosis and the more aggressive behavior of prostate tumors in obeses should be investigated.
\end{abstract}

Keywords: BMI; Gleason grade; Obesity; Prostate Biopsy; Prostate cancer

\section{Introduction}

Several studies investigated the correlation between obesity and prostate cancer with inconsistent results. A lower prostate cancer incidence has been reported in obese men aged 65 or less $[1,2]$. On the other hand, a fair evidence emerges that prostate tumors in obese men have high-grade disease, more aggressive behavior and worse prognosis $[3,4]$. Body Mass Index (BMI), is a worldwide adopted and cost-effective method to measure overall adiposity, although is it does not account for body mass composition and fat tissue distribution.

Higher BMI is associated with lower PSA (Prostatic Specific Antigen) plasmatic levels, that are $7 \%$ lower in overweight patients (BMI:25-29), 14\% lower in obese patients (BMI:30-35), and 18\% lower in severely obese patients (BMI>35) [5]. Consequently, a detection bias might be responsible of later diagnosis and more advanced T-stage and Gleason grade [6,7].

Gleason score is the most widely adopted grading system for prostate carcinoma. It is based on the sum of two patterns (or grades) ranging from 1 to 5 , with 5 having the worst prognosis. The first number is the grade of the most common tumor pattern, the second number is the grade of the second most common pattern. The Gleason Score ranges from 2 to 10 , with 10 having the worst prognosis.

In few studies, obesity has been related with higher Gleason grade and when compared with normal weight population [8]. Increasing proportion of Gleason pattern 4 and/or 5 is a critical factor in predicting biochemical recurrence, progression and mortality of prostate cancer.

The endocrine activity of visceral fat could be responsible of an increase of biological factors interacting with the differentiation of the prostatic malignant cell towards a more aggressive fenotype. High plasmatic levels of adipokines have been related to higher Gleason scores in obese patients. Leptin stimulates prostate cell proliferation, migration, growth factor expression and androgen resistance. Adiponectin plays opposite role. Prostate gland is exposed both to circulating adipokines and to those locally produced by periprostatic fat. It is plausible that abnormal levels of adipokines, interacting with androgens and other factors might select cells with a higher aggressiveness, in an early phase of metabolic syndrome when obesity is not yet relevant.

If a higher incidence of Gleason pattern 4 at biopsy would be confirmed in obese and overweight men compared to normal population, this finding would explain their worse prognosis suggesting the need for more aggressive diagnostic approach not delaying biopsy and adopting more extensive bioptic templates.

The aim of our study was to correlate obesity, measured by Body Mass Index (BMI), with the presence of Gleason pattern 4 or higher at biopsy.

\section{Materials and Methods}

Consecutive patients with positive prostate biopsy performed for palpable prostate nodule and/or elevated PSA levels were entered in our study. A written informed consent was obtained in all patients. BMI was adopted to classify patients according to obesity. Patients were divided into four categories according to BMI as follows: 16-19,9 (underweight), 20,0-24,9 (normal weight), 25,0-29,9 (overweight) and $\geq 30,0$ (obese).

*Corresponding author: Vincenzo Serretta, Department of Surgical, Oncological and Stomathological Sciences, University of Palermo, Italy, E-mail: vserretta@libero.it

Received July 09, 2013; Accepted August 30, 2013; Published September 02, 2013

Citation: Serretta V, Caruana G, Sommatino F, Scurria S, Carità G, et al. (2013) Does Exist A Correlation Between BMI and Gleason Patterns 4 and 5 At Prostate Biopsy? J Cytol Histol 4: 182. doi: 10.4172/2157-7099.1000182

Copyright: @ 2013 Serretta V, et al. This is an open-access article distributed under the terms of the Creative Commons Attribution License, which permits unrestricted use, distribution, and reproduction in any medium, provided the original author and source are credited. 
A transrectal prostate biopsy procedure with 12 cores, was performed in all patients.

The number of cores was increased in case of re-biopsy (18-24 cores or more).

All the specimens were reviewed by an expert pathologist.

Statistics: The statistical analysis was conducted with Fisher's exact test for Gleason pattern $4(<4$ or $\geq 4)$ and BMI for single weight class. The Pearson's Chi-squared test with Yates' continuity correction was used for aggregate BMI classes. A P value of $<0.05$ was considered statistically significant.

\section{Results}

Out of 164 patients diagnosed with prostate cancer at biopsy, 29 (17.7\%) were excluded from the study. Particularly, ASAP (Atypical Small Acinar Proliferation) or PIN (Prostatic Intraepithelial Neoplasia) was diagnosed in 5 , a single microscopic positive foci with undetermined Gleason score in 6 and a biopsy with a low number of bioptic cores, between 4 and 6 , not evaluable for the aims of our study was obtained in the remaining 18 patients.

Of the 135 evaluable patients, $20(14,81 \%)$ patients had a previous negative biopsy.

Patients characteristics are reported in Table 1.

The median age was 71 years (range 49-76). The median BMI was 26.8 (17.5-37.4). Only 2 patients (1,48\%) were underweight. 38 $(28,15 \%)$ patients had normal weight (median BMI 23,15), 61 (45,18\%) were overweight (median BMI 27,3) and $34(25,18 \%)$ were obese (median BMI 31,7)

Median PSA was $10,55 \mathrm{ng} / \mathrm{ml}$ (range $0,41-1339$ ). A prostate nodule was palpable in $55(40,7 \%)$ patients. The median prostate volume was 40,4 cc. (Table 1). A Gleason pattern 4 or 5 was evident in 57 $(42,2 \%)$ patients, while it was not detected in $78(57,8 \%)$ patients. The distribution of Gleason patterns according to BMI classes are given in Figure 1.

The statistical analysis did not demonstrate a correlation between the histological expression of Gleason pattern 4 or 5 in the bioptic specimens and the BMI class $(\mathrm{p}=0,79)$. Even combining different BMI

\begin{tabular}{|c|c|}
\hline $\begin{array}{l}\text { ENTERED PATIENTS } \\
\text { EVALUABLE PATIENTS }\end{array}$ & $\begin{array}{l}164 \\
135\end{array}$ \\
\hline MEDIAN AGE (years) & $71(49-86)$ \\
\hline \multicolumn{2}{|l|}{ BMI CLASSES } \\
\hline underweight & $2(1.48 \%)$ \\
\hline normal weight & $38(28.15 \%)$ \\
\hline over weight & $61(45.18 \%)$ \\
\hline obese & $34(25.18 \%)$ \\
\hline MEDIAN BMI (Kg/m²) & $26.8(17.5-37.4)$ \\
\hline underweight & 18.13 \\
\hline normal weight & 23.15 \\
\hline over weight & 27.3 \\
\hline obese & 31.7 \\
\hline MEDIAN PSA (ng/ml) & $5(0.41-1339)$ \\
\hline POSITIVE DRE & $55(40.7 \%)$ \\
\hline MEDIAN PROSTATE VOLUME (CC) & $40.4(18-24)$ \\
\hline PREVIOUS BIOPSY & $20(14.81 \%)$ \\
\hline
\end{tabular}

Table 1: Patients' characteristics and their distribution according BMI classes and median BMI.

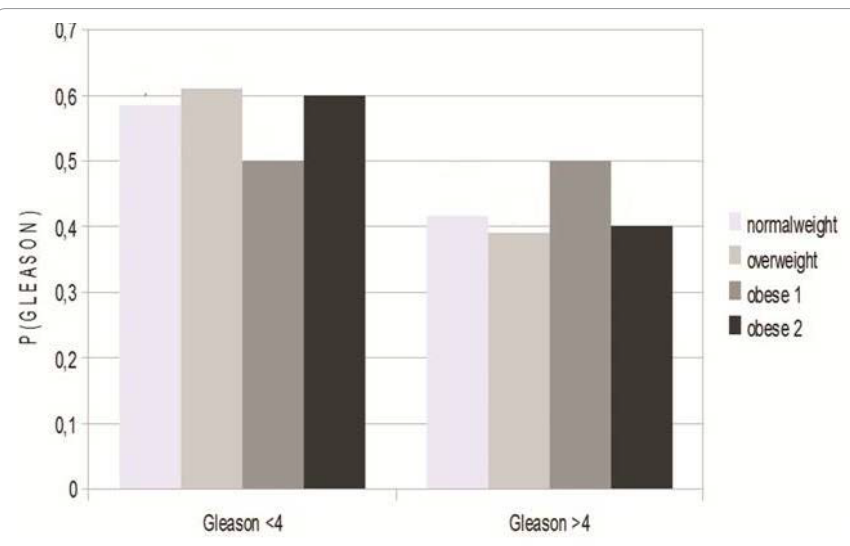

Figure 1: Correlation between the expression of Gleason pattern 4 and 5 and BMI:

No difference emerges in Gleason distribution in relation with the BMI.

\begin{tabular}{|l|l|}
\hline EVALUABLE PATIENTS & 135 \\
\hline Gleason pattern $\mathbf{4}$ or $\mathbf{5}$ & $57(42.2 \%)$ \\
\hline Gleason patterns $\mathbf{4 / 5}$ and BMI & $\mathrm{p}=0.79$ \\
\hline Normal vs Overweight + Obese & $\mathrm{p}=0.64$ \\
\hline Normal + Overweight vs Obese & $\mathrm{p}=0.93$ \\
\hline
\end{tabular}

Table 2: Correlation between BMI classes and the occurrence of Gleason patterns 4 and 5 prostate tumors.

classes, normal weight plus overweight men versus obese $(p=0,64)$; normal weight versus overweight plus obese men $(p=0,93)$, no statistically significant relation emerged (Table 2).

\section{Discussion}

Biological and clinical evidence supports the presence of Gleason patterns 4 and 5 as relevant predictors of aggressive prostate cancer [9]. The patients harboring prostate cancer showing Gleason patterns 4 or 5 have a poorer prognosis and need early diagnosis and treatment. To select for biopsy procedure patients at risk for aggressive tumor avoiding diagnosis of indolent, low-risk prostatic cancer is a major challenge for the urologic and scientific community.

The distinct behavior between pattern 3 and pattern $4 / 5$ prostatic tumors could be the result of different developmental pathways, although up today is unclear which molecular changes are involved [10]. Presumably, environmental and hormonal factors can induce additional oncogenic mutations in genetically susceptible and instable prostate cells. When examined by stage and grade, obese men are more likely to be diagnosed with high grade disease $[3,7]$.

A link between high grade prostate cancers and lower serum testosterone concentrations in obese patients has been suggested by many Authors $[8,11]$. Moreover abnormal levels of adipokines, biological factors with relevant carcinogenetic activity produced by the visceral fat tissue, may promote the progression of latent or microscopic low grade prostate tumors to high grade clinically significant cancers $[12,13]$. Freedland and coll in 2008 [14] and more recently De Nunzio and coll [3] reported a frequent incidence of high Gleason grade at biopsy. This finding has important clinical implications since the treatment choice is mainly based, although not exclusively, on Gleason grade.

The aim of our study was to confirm the data of literature reporting higher Gleason grade, with prevalence of pattern 4 and 5, in obese 
patients and to investigate the relation between BMI and histology at prostate biopsy. If this was the case, a particular attention should be given to patients with higher BMI and a clinical suspect of prostate cancer. BMI might be considered together with the other prognostic factors suggesting the need for prostate biopsy. An increased number of biopsy cores has been advocated by some Authors in obese and overweight men due to an increased difficulty and delay in cancer detection. We included consecutive patients with a positive prostate biopsy for cancer. Mean PSA was $10 \mathrm{ng} / \mathrm{ml}$, median age 71 years and, noteworthy, almost half of the patients was overweight and more than $20 \%$ obese. This is, nowadays, a common finding in everyday clinical practice, in patients with a positive prostate biopsy when you are not selecting for radical treatments [15]. In our experience no statistically significant relations was found between patients' BMI and the distribution of Gleason patterns 4 and 5. Consequently, we cannot support BMI as a predictive factor of high grade disease suggesting the need for immediate prostate biopsy when a cancer is clinically suspected. A main bias of our study is the limited patients' number not permitting data stratification according to stage and PSA levels. On the other hand, unselected consecutive patients of everyday clinical practice, undergoing biopsy for different clinical queries, were included, giving a practical value to our results. Since more than $70 \%$ of our patients were overweight or obese, our negative results may not have been due to a lack of association between BMI and Gleason pattern 4 or 5, but rather to a homogeneous study population. It must be argued that many factors other than Gleason grade might be responsible of the poor outcome related to high BMI.

\section{Conclusion}

Most of the patients undergoing prostate biopsy in western countries are overweight or obese. Although high risk prostate cancer has been reported to be more frequent in patients with higher BMI, in our experience, no significant correlation between BMI and the expression of Gleason patterns 4 and 5 at biopsy was detected. Thus, BMI cannot be considered a prognostic factor for high grade disease to select patients for prostate biopsy. Other biological and clinical factors could be responsible of the aggressive behavior of the prostate tumors in overweight and obese patients and should be the object of future investigations.

\section{Acknowledgement}

We wish to thank the GSTU Foundation for the support

\section{References}

1. Giovannucci E, Rimm EB, Liu Y, Leitzmann M, Wu K, et al. (2003) Body mass index and risk of prostate cancer in U.S. health professionals. J Natl Cancer Inst 95: 1240-1244.

2. Porter MP, Stanford JL (2005) Obesity and the risk of prostate cancer. Prostate 62: 316-321.

3. De Nunzio C, Freedland SJ, Milano R (2011) Metabolic syndrome is associated with high grade Gleason score when prostate cancer is diagnosed on biopsy. Prostate 71: 1492-8.

4. Freedland SJ, Aronson WJ, Kane CJ (2004) Impact of obesity on biochemical control after radical prostatectomy for clinically localized prostate cancer: a report by the Shared Equal Access Regional Cancer Hospital database study group. J Clin Oncol 22:446-453.

5. Bañez LL, Hamilton RJ, Partin AW, Vollmer RT, Sun L, et al. (2007) Obesityrelated plasma hemodilution and PSA concentration among men with prostate cancer. JAMA 298: 2275-2280.

6. Freedland SJ, Platz EA, Presti Jr JC (2006) Obesity,serum prostate specific antigen and prostate size: implications for prostate cancer detection. J. Urol 175:500-4.

7. Allott EH, Masko EM, Freedland SJ (2013) Obesity and prostate cancer: weighing the evidence. Eur Urol 63: 800-809.

8. Hoffman MA, DeWolf WC, Morgentaler A (2000) Is low serum free testosterone a marker for high grade prostate cancer? J Urol 163: 824-827.

9. Trock BJ, Guo CC, Gonzalgo ML, Magheli A, Loeb S, et al. (2009) Tertiary Gleason patterns and biochemical recurrence after prostatectomy: proposal for a modified Gleason scoring system. J Urol 182: 1364-1370.

10. Lavery HJ, Droller MJ (2012) Do Gleason patterns 3 and 4 prostate cancer represent separate disease states? J Urol 188: 1667-1675.

11. Schatzl G, Madersbacher S, Thurridl T, Waldmüller J, Kramer G, et al. (2001) High-grade prostate cancer is associated with low serum testosterone levels. Prostate 47: 52-58.

12. Rowlands MA, Gunnell D, Harris R, Vatten LJ, Holly JM, et al. (2009) Circulating insulin-like growth factor peptides and prostate cancer risk: a systematic review and meta-analysis. Int J Cancer 124: 2416-2429.

13. Li H, Stampfer MJ, Mucci L, Rifai N, Qiu W, et al. (2010) A 25-year prospective study of plasma adiponectin and leptin concentrations and prostate cancer risk and survival. Clin Chem 56: 34-43.

14. Freedland SJ, Wen J, Wuerstle M, Shah A, Lai D, et al. (2008) Obesity is a significant risk factor for prostate cancer at the time of biopsy. Urology 72: 11021105.

15. Ly D, Reddy CA, Klein EA, Ciezki JP (2010) Association of body mass index with prostate cancer biochemical failure. J Urol 183: 2193-2199. 